\title{
Flattened loose particles from numerical simulations compared to particles collected by Rosetta
}

\author{
J. Lasue ${ }^{1}$, I. Maroger ${ }^{1}$, R. Botet ${ }^{2}$, Ph. Garnier ${ }^{1}$, S. Merouane ${ }^{3}$, Th. Mannel ${ }^{4,5}$, \\ A. C. Levasseur-Regourd ${ }^{6}$, and M. S. Bentley ${ }^{7}$
}

${ }^{1}$ IRAP, Université de Toulouse, CNRS, CNES, UPS, Toulouse, France e-mail: jlasue@irap.omp.eu

${ }^{2}$ LPS, Université Paris-Saclay/Université Paris-Sud/CNRS, UMR 8502, Orsay, France

${ }^{3}$ Max Planck Institute for Solar System Research, Göttingen, Germany

${ }^{4}$ Space Research Institute of the Austrian Academy of Sciences, Graz, Austria

${ }^{5}$ University of Graz, Graz, Austria

${ }^{6}$ CNRS, LATMOS, Sorbonne Université, Paris, France

${ }^{7}$ European Space Astronomy Centre, Madrid, Spain

Received 3 December 2018 / Accepted 22 February 2019

\begin{abstract}
Context. Cometary dust particles are remnants of the primordial accretion of refractory material that occurred during the initial formation stages of the solar system. Understanding their physical structure can help constrain their accretion process.

Aims. The in situ study of dust particles that were collected at slow speeds by instruments on board the Rosetta space mission, including GIADA, MIDAS, and COSIMA, can be used to infer the physical properties, size distribution, and typologies of the dust. Methods. We have developed a simple numerical simulation of aggregate impact flattening to interpret the properties of particles collected by COSIMA. The aspect ratios of flattened particles from simulations and observations are compared to distinguish between initial families of aggregates that are characterized by different fractal dimensions $D_{\mathrm{f}}$. This dimension can differentiate between certain growth modes: the diffusion limited cluster-cluster aggregates (DLCA, $D_{\mathrm{f}} \approx 1.8$ ), diffusion limited particle-cluster aggregates (DLPA, $D_{\mathrm{f}} \approx 2.5$ ), reaction limited cluster-cluster aggregates (RLCA, $D_{\mathrm{f}} \approx 2.1$ ), and reaction limited particle-cluster aggregates $\left(\mathrm{RLPA}, D_{\mathrm{f}} \approx 3.0\right.$ ).

Results. The diversity of aspect ratios measured by COSIMA is consistent with either two families of aggregates with different initial $D_{\mathrm{f}}$ (a family of compact aggregates with $D_{\mathrm{f}}$ close to $2.5-3$ and some fluffier aggregates with $D_{\mathrm{f}} \approx 2$ ) or aggregates formed by a single type of aggregation process, such as DLPA. In that case, the cohesive strength of the dust particles must span a wide range to explain the range of aspect ratios observed by COSIMA. Furthermore, variations in cohesive strength and velocity may play a role in the detected higher aspect ratio range $(>0.3)$.

Conclusions. Our work allows us to explain the particle morphologies observed by COSIMA and those generated by laboratory experiments in a consistent framework. Taking into account all observations from the three dust instruments on board Rosetta, we favor an interpretation of our simulations based on two different families of dust particles with significantly distinct fractal dimensions that are ejected from the cometary nucleus.
\end{abstract}

Key words. comets: general - protoplanetary disks - accretion, accretion disks - methods: numerical - space vehicles: instruments comets: individual: 67P/Churyumov-Gerasimenko

\section{Introduction}

Comets are believed to preserve pristine dust grains and to provide information about their aggregation processes in the early solar system (e.g., Weidenschilling 1997; Blum 2000). Analyses of data from the Giotto mission to comet 1P/Halley and of foil impacts and aerogel tracks retrieved by the Stardust mission in the coma of comet 81P/Wild 2 have indeed given clues to the presence of low-density dust particles that are built up of agglomerates, possibly with different tensile strengths and porosities (e.g., Fulle et al. 2000; Hörz et al. 2006; Burchell et al. 2008). The interpretation of remote polarimetric observations of bright comets such as 1P/Halley and C/1995 O1 Hale-Bopp has led to similar conclusions (Levasseur-Regourd et al. 2008; Lasue et al. 2009). Aggregation of solid particles in the early solar system may therefore form a diversity of porosities that is represented by their fractal dimension, $D_{\mathrm{f}}$ (Dominik \& Tielens 1997; Kempf et al. 1999; Bertini et al. 2009). Understanding the structure of cometary dust particles can give clues to these early Solar System processes (Blum \& Wurm 2008; Fulle \& Blum 2017).

During its 26-month-long rendezvous with comet 67P/Churyumov-Gerasimenko (hereafter 67P) in its 2015 apparition, the Rosetta spacecraft monitored the properties of cometary dust particles that were released by the nucleus in the pre- and post-perihelion phases, as well as during some outburst events. Three instruments were specifically devoted to the study of dust particles: (i) the COmetary Secondary Ion Mass Analyzer (COSIMA; Kissel et al. 2007) collected dust particles of $10-100 \mu \mathrm{m}$ size on $1 \mathrm{~cm}^{2}$ targets, imaged them with a microscope operating under grazing incidence illumination with a resolution of about $14 \mu \mathrm{m}$, and then analyzed them 
through a mass spectrometer after indium ion beam ablation, (ii) the Micro-Imaging Dust Analysis System, (MIDAS; Riedler et al. 2007) collected micron-sized dust particles on targets of about $3.5 \mathrm{~mm}^{2}$ in order to obtain 3D images of their surfaces down to a pixel resolution of tens of nanometers using atomic force microscopy, and (iii) GIADA (the Grain Impact Analyzer and Dust Accumulator, Colangeli et al. 2007) measured the optical cross-section, speed, momentum and cumulative flux of hundreds of submillimeter-sized dust particles.

The COSIMA and MIDAS instruments collected dust particles at velocities in the $1-15 \mathrm{~m} \mathrm{~s}^{-1}$ range (Fulle et al. 2015), that is to say at relative velocities much lower than the $6.1 \mathrm{~km} \mathrm{~s}^{-1}$ reached during the collection of $81 \mathrm{P} /$ Wild 2 samples. Their chemical properties were thus mostly preserved, as was part of their physical structure. Some small particles, which could be fragments of fragile individual particles, were still noted (e.g., Bentley et al. 2016; Merouane et al. 2016). Interestingly enough, some particles appeared to be flattened, most likely as a result of impact alteration (e.g., Langevin et al. 2016; Mannel et al. 2016).

The Rosetta dust experiments provide complementary insights into the properties of dust particles, which was made possible by their different approaches (for a review, see Levasseur-Regourd et al. 2018). The total number of dust particles detected in the images is above 30000 for COSIMA and above 1000 for MIDAS (Levasseur-Regourd et al. 2018; Güttler et al. 2019).

More specifically, all images of dust particles indicate that the particles consist of more or less porous agglomerates of smaller grains (following the classification introduced in Güttler et al. 2019). Their overall sizes, identified by well-defined boundaries, range from about $1 \mu \mathrm{m}$ to tens of micrometers for MIDAS, and from tens of micrometers to several hundreds of micrometers for COSIMA. The presence of aggregated structures at distinct scales suggests a hierarchical aggregation (Bentley et al. 2016). The fractal dimension of a very porous agglomerate detected by MIDAS was determined through a density-correlation function (Mannel et al. 2016) to be equal to $1.7 \pm 0.1$. Dust showers observed by GIADA were also explained by the presence of fragile agglomerates with a fractal dimension $D_{\mathrm{f}}<2$, possibly disrupted through electrostatic fragmentation induced by the spacecraft (Fulle et al. 2015, 2016). Considering fractal aggregation processes, the porosity of dust particles in 67P can thus be estimated to be at least equal to $90 \%$ for very porous particles and about $75 \%$ for more compact particles (e.g., Blum \& Wurm 2008; Bertini et al. 2009). The porosity of dust particles of $67 \mathrm{P}$ has been estimated to be around $60 \%$ based on the density of the nucleus and the composition measured by COSIMA (Fulle et al. 2017). Analysis of the reflectance of porous dust particles collected by COSIMA indicate that a high porosity $(>50 \%)$ is necessary to explain that the mean free path of photons in the particle correspond to a significant fraction of the particle size (Langevin et al. 2017).

Finally, it may be added that the properties of cometary dust particles as revealed by the Rosetta mission are, as previously suspected, remarkably comparable to chondritic porous interplanetary dust particles (CP-IDPs) collected in the Earth's stratosphere, and UltraCarbonaceous Antarctica MicroMeteorites (UCAMMs) collected in the snows of central regions of Antarctica (e.g., Levasseur-Regourd et al. 2018).

The morphology, structure, and composition of such dust particles strongly suggest that as the cometary nuclei themselves, they formed in the solar nebula and the primordial disk (e.g., Davidsson et al. 2016; Blum et al. 2017), and were never processed within large objects.

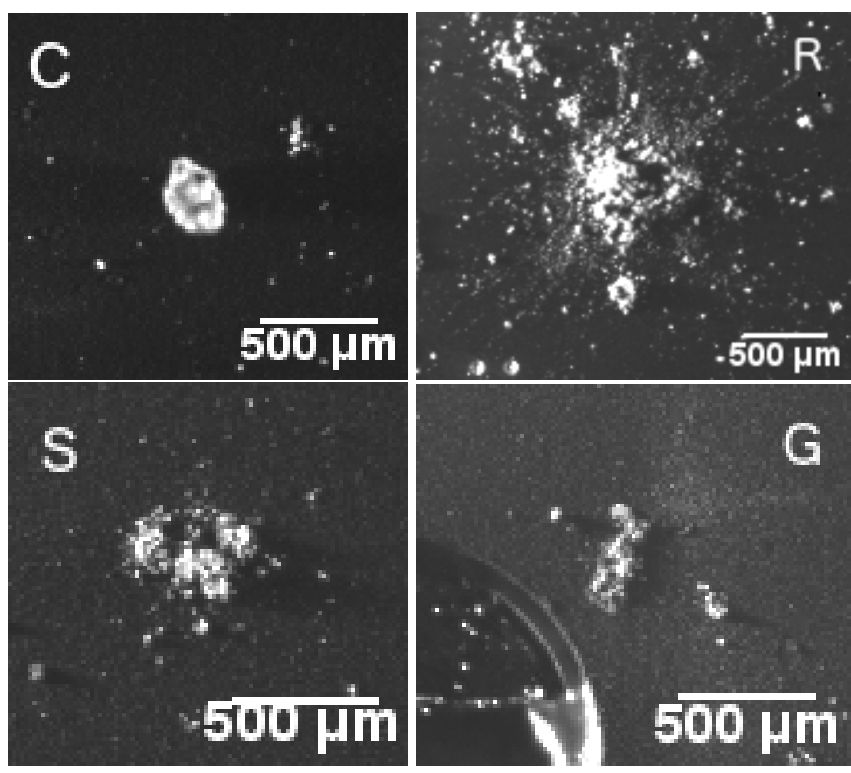

Fig. 1. Diversity of crushed particle types detected by COSIMA (Nick: compact particle, C; Alexandros: rubble pile, R; Estelle: shattered cluster, S; and Johannes: glued cluster, G; adapted from Langevin et al. 2016).

COSIMA collected and analyzed cometary particles ejected by 67P on gold black-covered targets (Kissel et al. 2007). The dust particles ejected by the comet impacted COSIMA targets at a speed $<10 \mathrm{~m} \mathrm{~s}^{-1}$ according to GIADA measurements (Rotundi et al. 2015), with a deceleration $<1 \times 10^{6} \mathrm{~m} \mathrm{~s}^{-2}$ according to Hornung et al. (2016). These values are enough to damage the initial structure of the dust particles during the collision, as visually assessed from the images acquired by COSISCOPE after collection. With a resolution of $14 \mu \mathrm{m}$, the microscope enabled studies of particle typology and flux (Langevin et al. 2016). The images show particles ranging from a few tens to several hundreds of microns, the majority of which appears to be built of micron-sized subcomponents, as confirmed by MIDAS (Bentley et al. 2016). Analysis of the particle morphologies identified four particle families (Langevin et al. 2016), which fall into two major classes, compact and clustered. These families are listed below.

1. Compact (type C) particles present well-defined boundaries without smaller satellite particles and with an apparent height above the collecting plane of the same order of magnitude as their horizontal ( $x$ and $y$ ) dimensions.

2. Shattered cluster (type $S$ ) particles are defined by clusters of fragments for which no individual fragment makes up a major fraction of the initial particle. These particles are interpreted as rearrangement of fragments within the impacting particle without associated disruption.

3. Glued cluster (type G) particles have a well-defined shape and a complex structure where sub-components appear to be linked by a fine-grained matrix with a smooth texture.

4. Rubble piles (type R) particles comprise components much smaller than their apparent size. Upon collision with the plate, the sub-components rearranged themselves in a flattened conical pile with many satellite components indicating poor cohesion.

The different particle types that were collected by COSIMA are illustrated in Fig. 1.

The grazing incidence illumination provided by COSISCOPE allows both the surface area of the collected particles and their height (based on their projected shadow) to be determined 


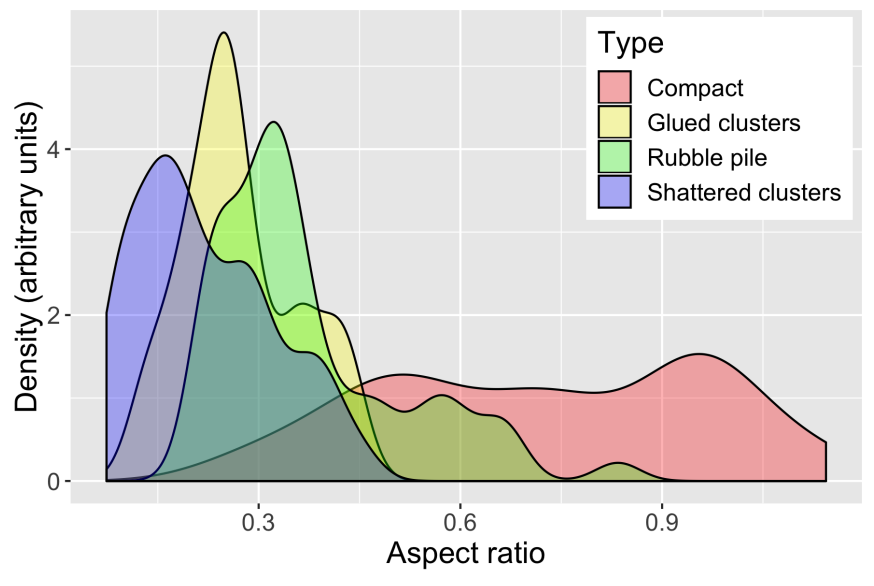

Fig. 2. Probability density of the aspect ratio for each type of particles detected by COSIMA (adapted from Langevin et al. 2016).

(see Fig. 1). The area is determined from the ratio of bright pixels before and after exposure to the dust flux from the comet. An aspect ratio of the compacted particles can be obtained from $\frac{\text { height }}{\sqrt{\text { area }}}$. The aspect ratio density distribution for each detected particle type is shown in Fig. 2. The compact particles, C, appear unbroken and present the highest aspect ratios, with a first peak around 0.5 and another close to 1 . The other particles present typical aspect ratios of around 0.3, and the shattered clusters are the flattest type of agglomerates. To understand the physical structure of cometary nuclei, it is important to infer, as far as possible, properties of dust particles prior to their collection. COSIMA analyses have shown a correlation between the flux of dust particles at various distances from the comet nucleus and their morphology (Merouane et al. 2016). The fragmenting particles appear to have a mechanical strength of a few $1000 \mathrm{~Pa}$ (Hornung et al. 2016) and their morphological diversity could result from different collection speeds in the range from $1 \mathrm{~m} \mathrm{~s}^{-1}$ to $6 \mathrm{~m} \mathrm{~s}^{-1}$ as investigated by laboratory simulations (Ellerbroek et al. 2017).

In this work, we investigate if different dust particle structures prior to their collection can also lead to the different morphologies found by the Rosetta dust instruments. We present a set of numerical simulations of fractal aggregates that flatten on impact with a plane surface, before we present the results and discuss their implications for the interpretation of the Rosetta measurements.

\section{Method}

\subsection{Fractal aggregate models}

We expect the dust particles that aggregate in the solar nebula to present fractal structures. Fractal aggregates in the early solar system form a diversity of porosities that can be represented by their fractal dimension, $D_{\mathrm{f}}$, based on their aggregation processes (Wurm \& Blum 1998). Aggregation simulations consider the collisions of spherical monomers which represent individual grains aggregating to form dust particles (Güttler et al. 2019). Four main aggregation processes that lead to significantly different fractal dimensions are considered: DLCA $\left(D_{\mathrm{f}} \approx 1.8\right)$, RLCA $\left(D_{\mathrm{f}} \approx 2.1\right)$, DLPA $\left(D_{\mathrm{f}} \approx 2.5\right)$, and RLPA $\left(D_{\mathrm{f}} \approx 3\right)$. The DL models are diffusion limited models, in which one monomer directly sticks to another when they meet. The RL models are reaction limited models, in which molecular reactions occur when two monomers encounter each other and slide with respect to one another so that the number of bonds is maximized as they form a more compact aggregate. CA stands for cluster-cluster aggregation, and PA for particle-cluster aggregation: in PA particles, monomers are added to the same main cluster that accretes all the mass and is relatively compact, whereas in CA particles, monomers form separate clusters that then aggregate, thus resulting in a smaller fractal dimension of the aggregate. The PA process occurs when the number of monomers in the available volume is high, which increases the possibility of collision among small aggregates.

Depending on the physical conditions of the primordial protosolar nebula, in terms of dust-to-gas ratio and dust composition, we can expect each of these types of aggregates to be formed (Weidenschilling 1997; Kimura 2001). They have also each been produced by computer simulations and laboratory experiments that simulated the initial stages of planetary accretion (Meakin 1991; Blum \& Wurm 2008).

\subsection{Flattening simulation}

In a first step, 3D off-lattice aggregates of a number $N=10000$ identical spherical particles (called monomers) were generated according to the four different aggregation processes described above. The resulting fractal aggregates were characterized by different initial fractal dimensions according to the approximate relationships DLCA $\left(D_{\mathrm{f}} \approx 1.8\right)$, RLCA $\left(D_{\mathrm{f}} \approx 2.1\right)$, DLPA $\left(D_{\mathrm{f}} \approx 2.5\right)$, and RLPA $\left(D_{\mathrm{f}} \approx 3\right)$. The values were calculated using the well-known self-similarity properties of fractals, whereby the number, $N_{\mathrm{m}}$, of monomers constituting the aggregate located within a sphere of radius $R$ follows $N_{\mathrm{m}} \propto R^{D_{\mathrm{f}}}$, where $R$ is smaller than the gyration radius of the aggregate. The gyration radius of a fractal aggregate is a measure of the extent of the aggregate, similar to the standard deviation of the monomers' distance to the center of mass of the aggregate and can be calculated by

$R_{\mathrm{g}}^{2}=\frac{1}{2 N} \times \sum_{i, j}\left(r_{i}-r_{j}\right)^{2}=\frac{1}{N} \times \sum_{i}\left(r_{i}-r_{\mathrm{c}}\right)^{2}$,

where $N$ is the number of monomers in the aggregate, $r_{i}$ and $r_{j}$ are the spatial coordinates of the center of the monomers $i$ and $j$, and $r_{\mathrm{c}}$ corresponds to the spatial coordinates of the center of mass of the aggregate (Jullien \& Botet 1987). A representation of each of the four aggregate types is given in Fig. 3. These aggregates may correspond to different types of cometary particles as ejected from the surface of the nucleus by gas pressure. For each aggregate type, 1000 different aggregation simulations were performed to statistically analyze the results.

In a second step, simulating the particle collection and flattening observed by COSIMA during the Rosetta mission, the aggregates were projected monomer by monomer onto the plane $z=0$, as shown in Fig. 4. The monomers were selected iteratively by increasing $z$ values. When a monomer was projected directly onto the plane $z=0$ without encountering any other monomer, it stuck directly to the collision plane. When it encountered a monomer that was previously stuck below it, we considered that a bond existed between the two monomers and that it was broken if $E_{\mathrm{VdW}}<E_{\mathrm{k}}$ where $E_{\mathrm{VdW}}$ is the van der Waals energy and $E_{\mathrm{k}}$ is the kinetic energy of the incoming particle. This condition can be written as in Eq. (2) considering van der Waals interactions between the two spherical elements,

$$
\begin{aligned}
& E_{\mathrm{VdW}}=\frac{A_{\mathrm{H}} r}{12 d} \leq E_{\mathrm{k}}=\frac{2}{3} \pi r^{3} \rho(\sin \theta)^{2} V^{2}, \\
& \left(\sin \theta_{0}\right)^{2}=\frac{A_{\mathrm{H}}}{8 \pi r^{2} \rho d V^{2}}
\end{aligned}
$$



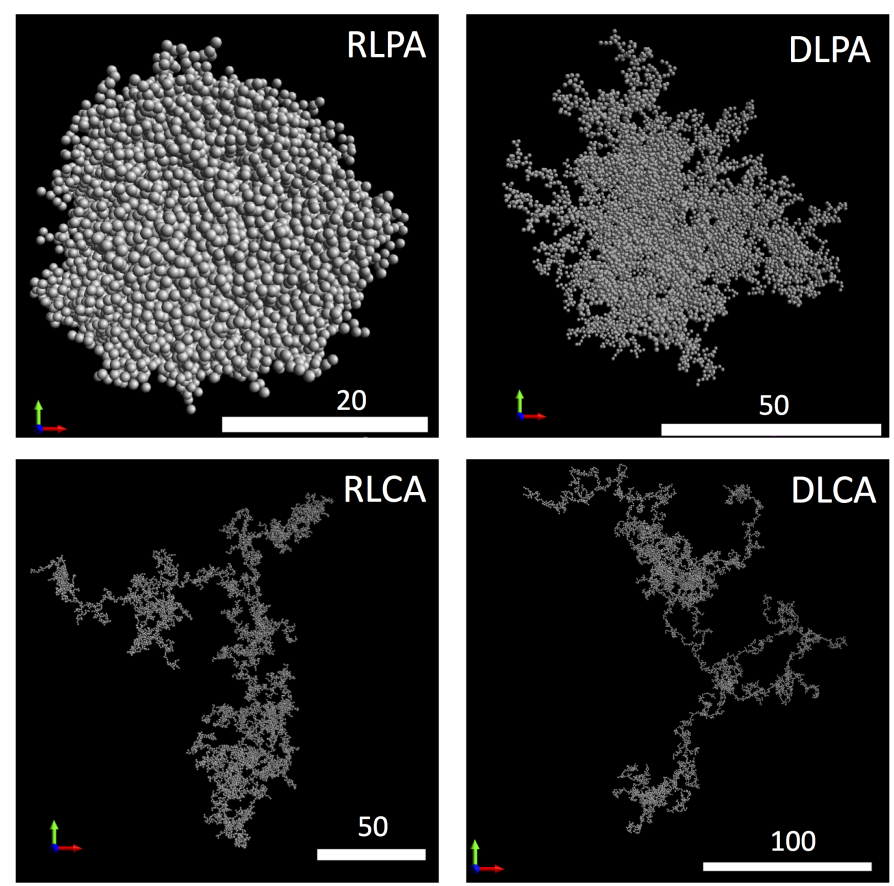

Fig. 3. 3D representation of four aggregates representing the four different aggregation processes considered in this work. DLCA $\left(D_{\mathrm{f}} \approx 1.8\right)$, RLCA $\left(D_{f} \approx 2.1\right)$, DLPA $\left(D_{\mathrm{f}} \approx 2.5\right)$, and RLPA $\left(D_{\mathrm{f}} \approx 3\right)$. A scale is given in number of monomers, and a referential frame is indicated by colored arrows $(\mathrm{x}=$ blue, $\mathrm{y}=$ red, and $\mathrm{z}=$ green $)$.

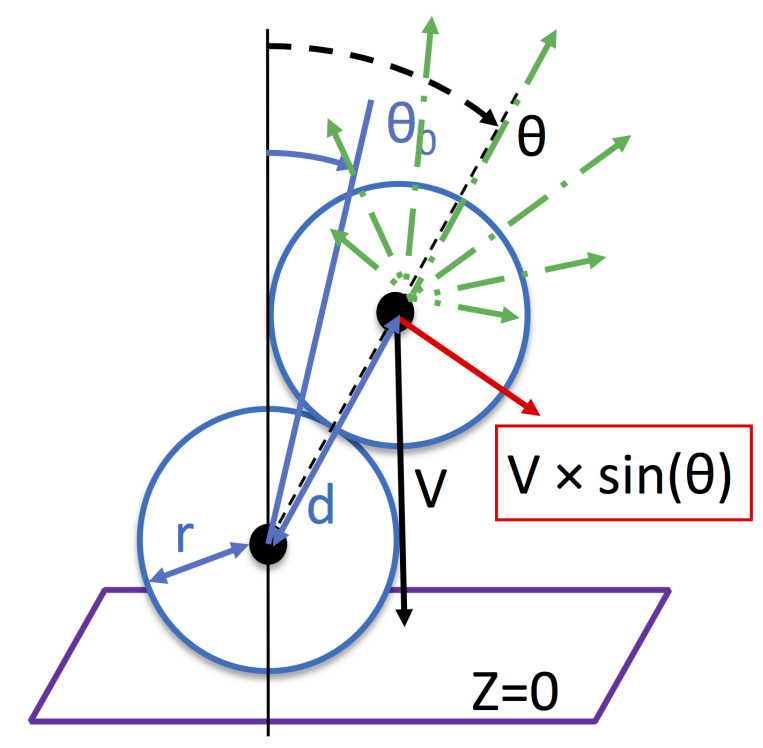

Fig. 4. Representation of the collision geometry for two superposed monomers. When $\theta<\theta_{0}$, then the superposition remains stable. When $\theta>\theta_{0}$, the cohesive link between the monomers is broken and the upper monomer will bounce randomly following some of the green arrows and will attach itself to the $z=0$ surface at a further point, thus fragmenting the aggregate.

where $E_{\mathrm{VdW}}$ is the van der Waals energy, $A_{\mathrm{H}}$ is the Hamaker constant for the material considered, $r$ is the radius of a single monomer, $d$ is the diameter of a monomer, $E_{\mathrm{k}}$ is the kinetic energy of the monomer, $\rho$ is the density of the monomer, $\theta$ is the angle between the direction linking the two centers of the monomers and the vertical direction, as illustrated in Fig. 4, and $V$ is the velocity of the aggregate with respect to the collecting surface $z=0$. The monomer diameter, $d$, is slightly larger (by $0.4 \mathrm{~nm}$ ) than the steady-state distance between the centers of two touching monomers. Typical monomer diameters are considered to be $20 \mathrm{~nm}$ or larger, making this difference negligible $(<2 \%)$. We thus considered the distance between two touching monomer centers to be equal to $d$. The Hamaker constant of two interacting particles corresponds to a measure of the relative strength of the particle material with respect to the attractive van der Waals forces between them (Hamaker 1937).

We call $\theta_{0}$ the angle $\theta$ for which Eq. (2) is an equality. $\sin \theta_{0}$ is a threshold above which monomers may break their bonds and bounce. Changing this parameter can either be viewed as changing the cohesive strength between monomers or as changing the collection velocity, as Eq. (3) shows. Thus, with the Hamaker constant of dry minerals under vacuum conditions $A_{\mathrm{H}} \approx 1 \times 10^{-19} \mathrm{~J}$ (Israelachvili 2011):

- $\sin \theta_{0} \approx 1$ corresponds to very cohesive monomer bonds, low collection speed, or very small monomer size (value typically obtained for $V=1 \mathrm{~m} \mathrm{~s}^{-1}$ and $r=0.01 \mu \mathrm{m}$ or for $A_{\mathrm{H}}$ values higher than $1 \times 10^{-19} \mathrm{~J}$ );

- $\sin \theta_{0} \approx 10^{-3}$ corresponds to all bonds being broken, relatively higher collection speed, or larger monomer sizes (value typically obtained for $V=10 \mathrm{~m} \mathrm{~s}^{-1}$ and $r=0.1 \mu \mathrm{m}$ ).

When a projected monomer meets another monomer, we can compute a collision parameter $\left(v=\frac{\sin \theta}{\sin \theta_{0}}\right)$. When $v \leq 1$, the monomer sticks to the monomer it bumps into. When $v>1$, the incoming monomer bounces according to a random direction based on the Lambertian reflection rule (see Fig. 4) and sticks to the plane $z=0$ or previously stuck monomers if they are present.

To make the model more realistic with respect to potential mass loss that may be incurred by the aggregates as they are flattened and their bonds are broken, in further simulations a mass-loss probability $P_{\text {loss }}$ was introduced. In this case, when a monomer meets condition $v>1$, then it was removed from the simulation with a mass-loss probability $P_{\text {loss }}$ that matches the probability that some monomers do not stick to any others and bounce back to free space during the collision.

An illustration of the effect of changing the value for $\sin \left(\theta_{0}\right)$ is given in Fig. 5, where the morphology of flattened aggregates is clearly dependent upon the initial structure of the aggregates and the geometric parameters. Under conditions where most bonds are broken $\left(\sin \theta_{0} \approx 10^{-3}\right)$, the more compact aggregates appear to generate a small pyramid of monomers with an angle of repose. The more porous the aggregates, the flatter the apparent projection. When bonds are unbroken $\left(\sin \theta_{0} \approx 1\right)$, similar structures appear, but some vertical chain-like columns of monomers extending upward are also present and increase the relative height of the flattened aggregate. These columns of monomers are due to the increased strength of the bonds between the monomers, forming chain-like vertical structures that are not broken by the flattening geometry (as $\theta_{0}>\theta$ over the monomers' column). We therefore see that parameters $\left(D_{\mathrm{f}}\right.$ and $\theta_{0}$ ) both significantly influence the outcome of the simulated projection.

Figure 6 shows the resulting projections when the monomers have a non-zero probability to bounce back to space as a result of mass-loss processes. In this case we only considered RLPA aggregates with different $P_{\text {loss }}$ values ranging from 0 to $50 \%$. With increasing mass-loss probability, only a flat footprint of the aggregate remains with a very low aspect ratio, which can represent the results of the low-speed laboratory aggregate sticking experiments of Ellerbroek et al. (2017), where most of the initial aggregate mass was lost. Such mass-loss processes may also be at work during the COSIMA particle collection. 


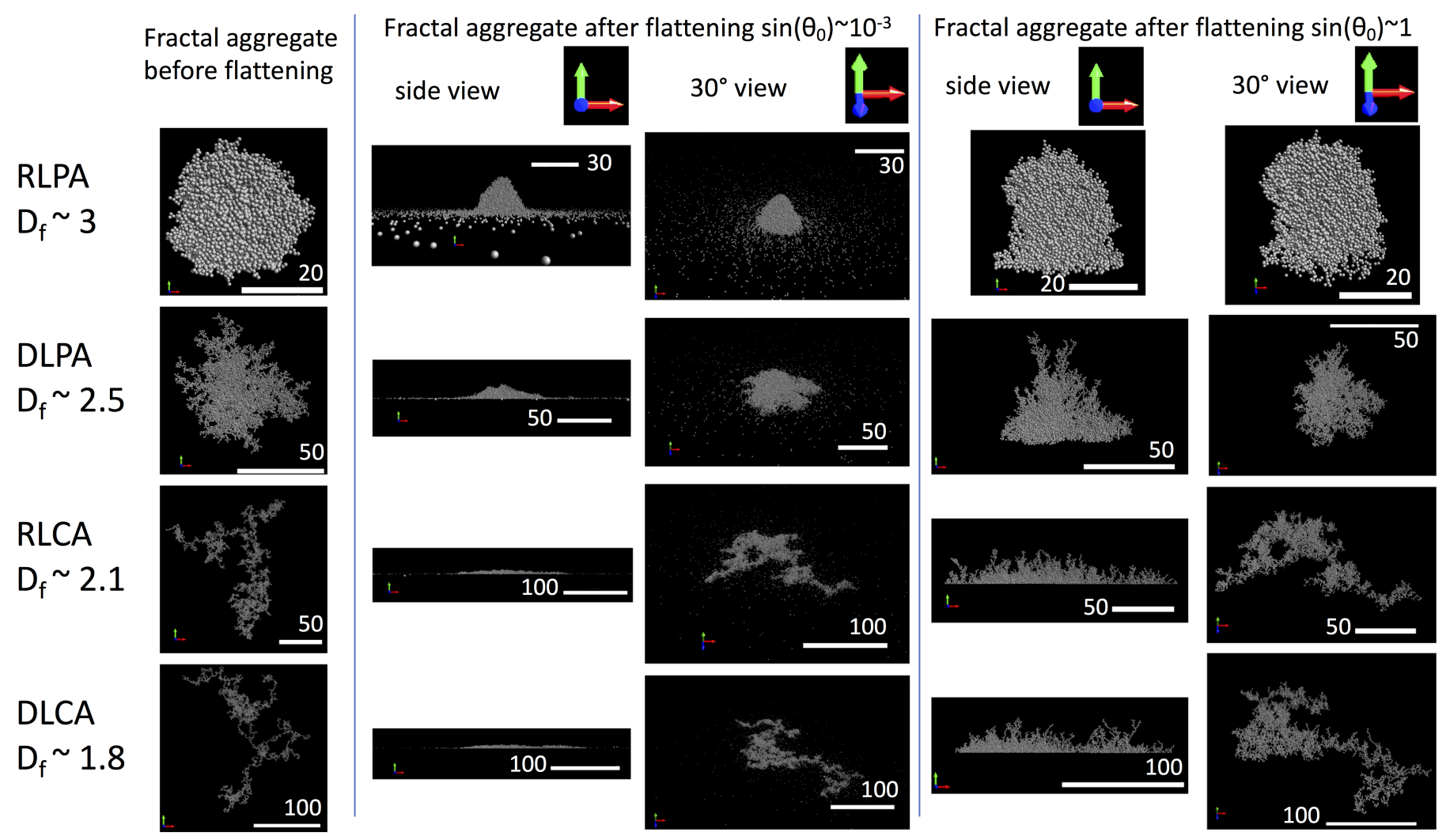

Fig. 5. Summary of the effect of the initial particle morphology $\left(D_{\mathrm{f}}\right)$ and bond cohesion on the morphology of the flattened particles. A scale is given in number of monomers, and a referential frame is indicated by colored arrows ( $\mathrm{x}=$ blue, $\mathrm{y}=\mathrm{red}, \mathrm{z}=$ green).
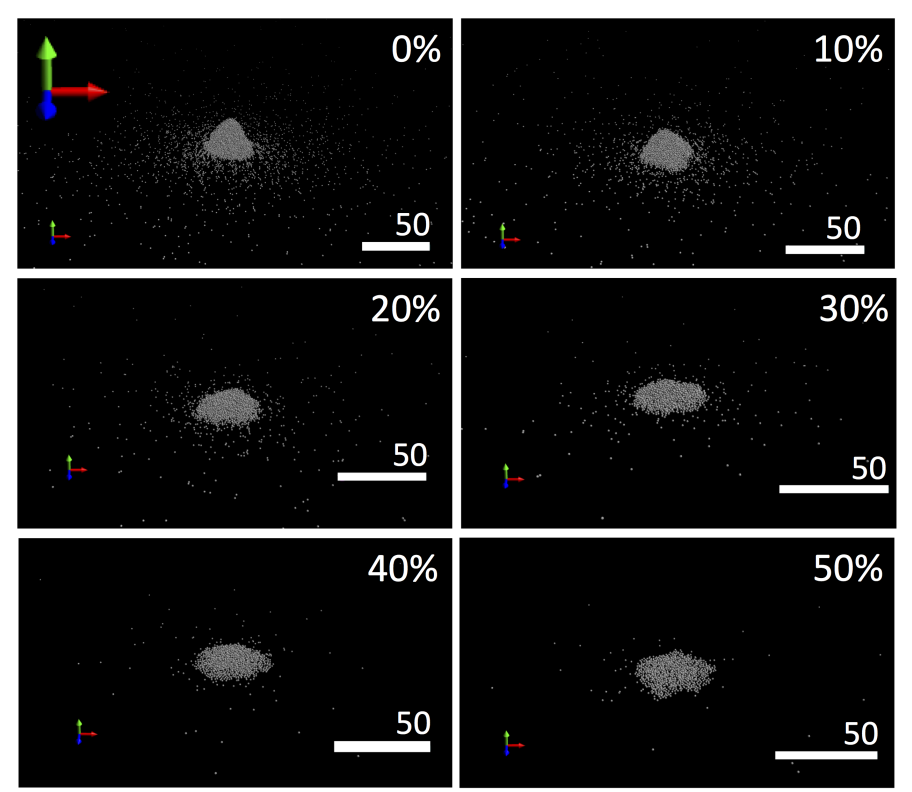

Fig. 6. 3D view of a RLPA aggregate after projection with different mass loss. A scale is given in number of monomers, and a referential frame is indicated by colored arrows $(\mathrm{x}=$ blue, $\mathrm{y}=\mathrm{red}$, and $\mathrm{z}=$ green $)$ with an approximate $30^{\circ}$ viewing angle.

\section{Results}

\subsection{Data analysis}

The aggregate flattening simulations were run to create 1000 aggregates of each of the four types, using 10000 monomers
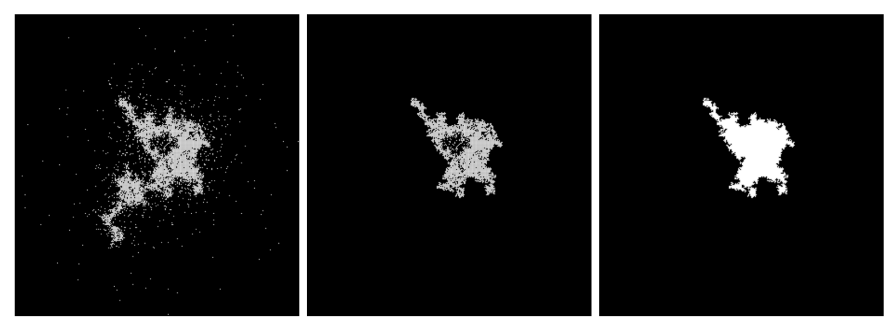

Fig. 7. Illustration of the connected area calculated for a flattened aggregate of type RLCA. The flattened particles seen from above is shown on the left. The calculated connected areas are shown with gaps in the middle and without gaps on the right. The parts of the aggregate that are not connected to the largest connected aggregate are removed from the processing.

each, for the four fractal dimensions considered; the $\sin \left(\theta_{0}\right)$ parameter ranged from 1 to $10^{-6}$ and the $P_{\text {loss }}$ mass-loss probability ranged from 0 to 0.5 . This was done in order to obtain good statistics for the aspect ratio of each numerically flattened aggregate for comparison to the COSIMA measurements. The height, $H$, of the flattened aggregates is the maximum value of $z$ of all the sticking monomers. To compute the area, $A$, we considered only those monomers that were visible from above (in the $-z$ direction), and based on their position, we calculated the contour of the projected connected monomer set (Lorensen \& Cline 1987). We computed two different connected areas: one with and one without gaps. They are shown in Fig. 7. The area with gaps was always somewhat smaller than the area without gaps, but it was essentially linearly correlated with it. Therefore, we calculated the results based on the connected area without gaps. In this way, we can calculate a statistical distribution of the aspect 
ratio, $H / \sqrt{A}$, for particles of each type, similar to the procedure used with the COSIMA data, and assess the effect of the different parameters on the morphology of the flattened aggregates.

\subsection{Morphologies of flattened aggregates}

Figure 8 represents the density distribution of aspect ratios calculated for the 1000 flattened aggregates of each fractal type and for four different values of $\sin \left(\theta_{0}\right)$. The upper figure is calculated for a $\sin \left(\theta_{0}\right)=1$ corresponding to a simulation where no bond between monomers is broken (illustrated on the righthand side of the Fig. 5). The distribution of the aspect ratios overlaps between about 0.5 (relatively flat aggregates) and 1.3. The distribution also separates the different types of aggregates relatively well; the more compact aggregates of type PA have a median aspect ratio value of $1.18(\sigma=0.15)$ and the fluffier aggregates of type CA have a median aspect ratio value of 0.73 $(\sigma=0.22)$. To first order, the process therefore appears to separate the aggregates with fractal dimensions $D_{\mathrm{f}}$ above or below 2 into two groups. This is somewhat expected because more compact aggregates will present more opportunities for solid vertical structures of monomers to remain unbroken and to vertically extend the projected aggregate. Moreover, the aspect ratio distributions of the CA type aggregates present an extended right wing, showing that some of these aggregates could still have aspect ratios close to one if their monomer bonds are stronger than the impact energy.

As $\sin \left(\theta_{0}\right)$ decreases, the number of broken bonds increases and the projected aggregates become flatter. The minimum aspect ratio decreases and reaches 0.1 for values of $\sin \left(\theta_{0}\right)=0.1$ or lower. The distribution of the most compact particles (RLPA with $D_{\mathrm{f}} \approx 3$ ) is now clearly separated from the distribution of the other aggregates and remains around 1, indicating that the surface dimensions covered by the flattened aggregate in $x$ and $y$ are of the same order of magnitude as its vertical extent in $z$. With respect to the distributions of the less compact aggregates, we note that the distributions for CA aggregates with fractal dimensions $D_{\mathrm{f}}<2$ quickly become undistinguishable. These flattened aggregates would therefore present essentially the same aspect ratio distributions, regardless of their initial morphology. The DLPA aggregates that have a fractal dimension around 2.5 are located in between these two extremes and are clearly separated from them at low values of $\sin \left(\theta_{0}\right)$. For example, the standard deviation of the distributions for $\sin \left(\theta_{0}\right)=0.25$ range from 0.06 to 0.09 . The DLPA distribution average aspect ratio is approximately 0.3 for $\sin \left(\theta_{0}\right)=0.1$ or lower. At values of $\sin \left(\theta_{0}\right)$ lower than 0.1 , the density distributions stabilize toward their final values. A bimodal density distribution for the flattened RLPA aggregates is also visible, which corresponds to whether vertical columns of monomers appear within the pyramid somewhat extending its height. We expect the random size distributions of monomers in real dust aggregates to limit the aspect ratio to the lower values of around 0.75-1.0. Some similar linear chainlike structures were also detected in the analysis of COSIMA particles, such as the 2CF Adeline particle (Hornung et al. 2016).

The effect of the $\sin \left(\theta_{0}\right)$ parameter is further illustrated in Fig. 9, where aspect ratio distributions of DLPA aggregates are calculated for different values of $\sin \left(\theta_{0}\right)$ ranging from 0.001 to 1 and are superposed. As the $\sin \left(\theta_{0}\right)$ value decreases, the aspect ratio decreases (because more bonds break) from approximately 1 to 0.25 . The standard deviation of the density distribution also decreases, indicating that most aggregates of this type flatten in the same way. This is related to the randomization of monomer deposition after bond breaking, which reduces the
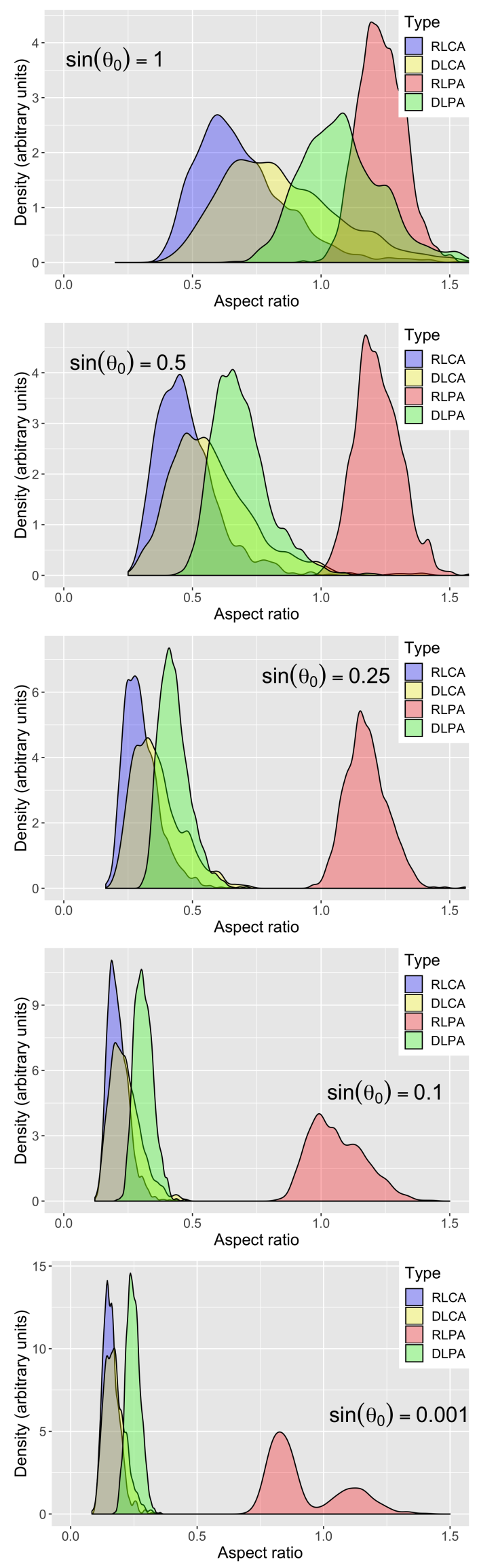

Fig. 8. Distribution of the aspect ratio, $H / \sqrt{A}$, for 1000 aggregates of each type (RLCA, DLCA, RLPA, and DLPA) with $\sin \left(\theta_{0}\right)$ ranging from 1 to 0.001 without mass loss. 


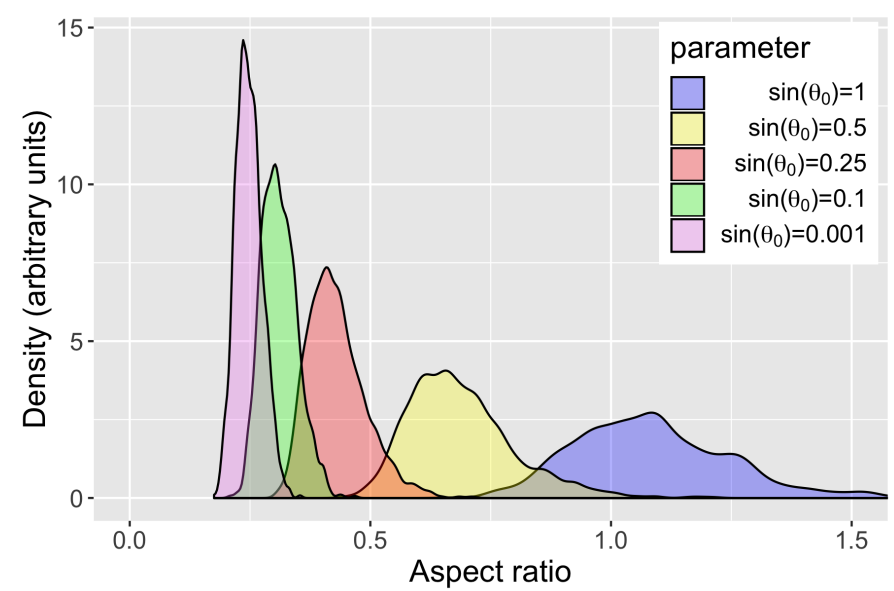

Fig. 9. Distribution of the aspect ratio, $H / \sqrt{A}$, for DLPA aggregates with different $\sin \left(\theta_{0}\right)$ without mass loss.

range of vertical extent that is possible after flattening. This figure shows that given a relatively narrow range of collection velocities, a wider range of bond cohesive strengths in the aggregates would lead to a wider range of aspect ratios for the same initial structure of the aggregate because Eq. (3) indicates that $\sin \left(\theta_{0}\right)$ is proportional to $\frac{1}{V}$. This is especially true of DLPAs because the aspect ratios for these particles range from 0.25 to 1.2. Compact aggregate aspect ratios would range between 0.8 and 1.3, while aggregates with fractal dimensions $D_{\mathrm{f}} \leq 2$ present aspect ratios ranging from 0.1 to 1 . When the cohesive strength of monomer bonds in the aggregate are randomly distributed, we expect to detect more aggregates with low flattened aspect ratios than large flattened aspect ratios.

Finally, the effect of the mass-loss coefficient is illustrated in the top panel of Fig. 10, where the probability density of aspect ratios for RLPA aggregates with $\sin \left(\theta_{0}\right)=1$ is calculated for mass-loss parameters ranging from 0 to $50 \%$. As expected, the mass-loss parameter reduces the aspect ratio of the flattened aggregates because of monomer loss. When we compare this to the variation in aspect ratio distribution for varying $\sin \left(\theta_{0}\right)$, the final aspect ratio distribution remains relatively wide (wider than 0.5 ), which is due to the simultaneous loss of monomers in all directions, so that the dimensions of the flattened aggregate are reduced in all dimensions at more or less the same rate (in $x$, $y$, and $z$ ). This parameter is also important in reducing the final aspect ratio of the flattened aggregates.

For DLPA and more fluffy aggregates, the initial aggregate is so porous that even moderate mass-loss destroys the structure during flattening. This rapidly leads to a very flat final projected structure, as illustrated in the bottom panel of Fig. 10.

It therefore appears that the initial fractal dimension of aggregates strongly affects the morphology of flattened aggregates, and that, it may be possible to distinguish the initial structure of the particles from their flattened morphologies depending on the effect of parameters such as the collection speed, the strength of bonds between the monomers, and the mass-loss fraction.

\section{Discussion}

\subsection{Comparison with COSIMA observations}

The aspect ratio variation with initial $D_{\mathrm{f}}$ (aggregate type), $\sin \left(\theta_{0}\right)$, and $P_{\text {loss }}$ can be compared with the values observed by
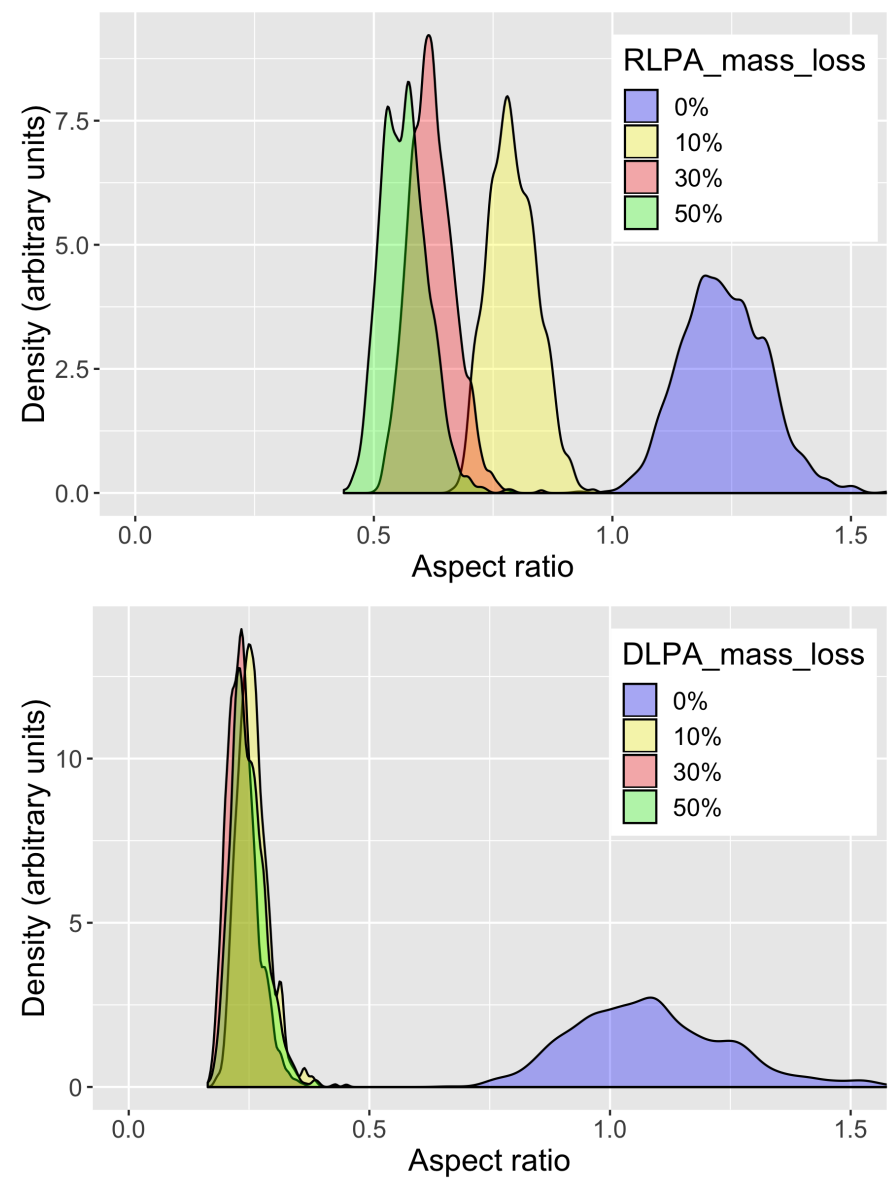

Fig. 10. Distribution of the aspect ratio, $H / \sqrt{A}$, for RLPA aggregates (top) and DLPA aggregates (bottom) with $\sin \left(\theta_{0}\right)=1$ and different massloss probability coefficients.

COSISCOPE that we present in Fig. 2. On the one hand, the aspect ratio of only the PA aggregate types is high enough to explain the presence of the compact particles in the COSIMA aspect ratio distribution. This implies that a population of particles with fractal dimension between 2.5 and 3 must be present in the distribution of particles ejected by 67P.

On the other hand, in order to explain the morphologies with aspect ratios as low as $0.1-0.3$, where the distributions of COSIMA particles of type $\mathrm{G}, \mathrm{R}$, and $\mathrm{S}$ peak, other types of particles or processes need to be invoked. According to our simulations, even with a mass loss as high as 50\%, RLPA aggregates alone cannot explain the range of observed aspect ratios. However, the DLPA type particles could reach aspect ratio values as low as 0.2 either with different cohesive strengths and/or velocities $\left(\sin \left(\theta_{0}\right)\right)$ or with mass losses of up to $50 \%$. Finally, a fractal dimension $D_{\mathrm{f}}<2$ would also lead to very low final aspect ratios even when particles with higher cohesive strengths are considered. The wide range of distributions observed by COSIMA could therefore be explained by the following scenarios:

1. two different initial groups of particles with low and high fractal dimensions (such as RLPA for the compact particles and DLPA for the shattered clusters).

2. The flattest kind of particles observed (shattered clusters with an aspect ratio around 0.15 ) could be consistent with compaction of the smallest fractal dimension RLCA and DLCA aggregates or with a very large mass loss during collection $(>50 \%)$. 
3. The diversity of morphologies could also originate from a single type of aggregation process (such as DLPA) but presenting very different cohesive strengths amongst aggregates $\left(\sin \left(\theta_{0}\right)\right.$ ranging from at least 0.1 to 1$)$. This distribution would also present a peak around 0.3 as shown in Fig. 9, which would be consistent with the peak of the COSIMA distribution around 0.3 as shown in Fig. 2.

4. Finally, a fourth process, described in Ellerbroek et al. (2017), may be playing a role here as well. Experiments show that incoming aggregates may sometimes fragment upon impact, leaving some remains sticking to the target in a pyramidal shape (mass transfer property between 0 and 0.8 ). The diversity of observed aspect ratios appears consistent with at least two families of aggregates with different $D_{\mathrm{f}}$, which would also be consistent with the GIADA and MIDAS measurements of two dust particle populations with very different fractal dimensions, one close to 3 and the other around 1.8 (Fulle \& Blum 2017; Mannel et al. 2016). Variations in both the cohesive strength of the particles and the speed of collection may play a role in the continuity of the higher aspect ratio range $(>0.3)$ detected by COSIMA. Alternatively, this could also mean that the initial low fractal dimensions have been somewhat altered by internal processes, such as compaction, or by temperature alteration, such as sintering, which may have occurred during the evolution of the cometary nucleus, especially on its surface.

\subsection{Comparison with collision experiments}

Ellerbroek et al. (2017) presented laboratory simulations of aggregate impacts that simulated the particle collection procedure of Rosetta. The aggregates were formed by aggregation of irregular polydisperse $\mathrm{SiO}_{2}$ particles with density around $2.6 \mathrm{~kg} \mathrm{~m}^{-3}$ and a size range of 0.1 to $10 \mu \mathrm{m}$. The final aggregates have porosities around $65 \pm 5 \%$ and low compressive strength between $1 \times 10^{4}$ and $1 \times 10^{6} \mathrm{~Pa}$. The aggregates were then accelerated by electrostatic forces toward a collecting plane where the collision was filmed and the resulting flattened footprint imaged and analyzed. The impact velocity ranged from about 1 to $6 \mathrm{~m} \mathrm{~s}^{-1}$.

The footprints represent the diversity of morphologies that were acquired by the COSIMA instrument. At very low velocities of around $1 \mathrm{~m} \mathrm{~s}^{-1}$, the aggregates either stick directly to the surface, similar to the compact COSIMA particle type, or they may bounce from the surface, leaving a very flat footprint with mostly unconnected fragments, which may be morphologically similar to the shattered cluster COSIMA type of particles. As velocities are increased from 2 to $6 \mathrm{~m} \mathrm{~s}^{-1}$, the particles mostly stick to the surface and fragmentation occurs, leading to footprints that are morphologically similar to COSIMA rubble piles or glued clusters.

In this laboratory work, all morphologies were generated using only a change in the impact velocity and impactor size, and similarities could be seen between the footprints of the particles that were obtained on the collecting surface and the morphologies measured by COSIMA. The simulations presented in our work allow us to generate similar flattening conditions by varying the velocity and the particle sizes. However, in our simulations, we can also modify the initial impacting particle morphology and study its effect on the flattening of the aggregates. This allows us to explore an extended set of parameters compared with the laboratory experiments, and we have shown that it is also possible to generate the measured footprint morphology by considering different initial fractal dimensions of the impacting particles, as discussed above. It would be of interest to study in the laboratory how very porous particles behave when subjected to the type of collection that occurred during the Rosetta mission to confirm our analysis.

\subsection{Possible analysis of MIDAS data}

A planned future study aims to investigate whether these results are also valid for MIDAS particles. The aspect ratios of dust particles collected by MIDAS should be calculated and their distribution reviewed. It will be of great interest if the distribution falls into different groups, and if they match those found in the simulation and with COSIMA particles. Because MIDAS particles are one order of magnitude smaller than those of COSIMA, this will allow us to understand how the initial structures of dust particles of comet 67P might look and if they remain similar over the $1-100 \mu \mathrm{m}$ size range.

\section{Conclusions}

We have shown that simple numerical simulations of aggregate flattening can be used to infer the initial properties of particles collected by COSIMA on-board Rosetta. The diversity of aspect ratios measured in COSIMA images appears consistent with several hypotheses on the initial properties of the collected particles.

1. It could be explained by at least two families of aggregates with different fractal dimensions $D_{\mathrm{f}}$. A mixture of some compact particles with fractal dimensions close to 2.53 together with some fluffier ones with fractal dimensions $<2$ would also be consistent with the observations and the measurements made by GIADA and MIDAS (Fulle \& Blum 2017; Mannel et al. 2016).

2. Alternatively, the distribution of morphologies seen by COSIMA could originate from a single type of aggregation process, such as DLPA $\left(D_{\mathrm{f}} \approx 2.5\right)$ but presenting a large range of cohesive strengths or collection velocities. This distribution would be consistent with a maximum at an aspect ratio around 0.3 as observed on the COSIMA typology (Langevin et al. 2016).

Furthermore, variations in cohesive strength and velocity may play a role in the higher aspect ratio range detected by COSIMA $(>0.3)$. Our work allows us to explain the particle morphologies observed by COSIMA and those generated by the laboratory experiments of Ellerbroek et al. (2017) in a consistent framework. Taken together with the observations made by GIADA and MIDAS on Rosetta, our simulations seem to favor the interpretation that two different families of dust particles with significantly distinct fractal dimensions were ejected from the cometary nucleus.

Acknowledgements. The authors acknowledge two anonymous referees for their positive evaluation and constructive comments. The authors acknowledge support from Centre National d'Etudes Spatiales (CNES) in the realization of instruments devoted to space exploration of comets and in their scientific analysis. T.M. acknowledges funding by the Austrian Science Fund FWF P 28100-N36.

\section{References}

Bentley, M. S., Schmied, R., Mannel, T., et al. 2016, Nature, 537, 73 Bertini, I., Gutierrez, P. J., \& Sabolo, W. 2009, A\&A, 504, 625

Blum, J. 2000, Space Sci. Rev., 92, 265

Blum, J., \& Wurm, G. 2008, ARA\&A, 46, 21

Blum, J., Gundlach, B., Krause, M., et al. 2017, MNRAS, 469, S755

Burchell, M. J., Fairey, S. A., Wozniakiewicz, P., et al. 2008, Meteorit. Planet. Sci., 43, 23 


\section{J. Lasue et al.: Flattened loose particles compared to Rosetta}

Colangeli, L., Lopez-Moreno, J. J., Palumbo, P., et al. 2007, Space Sci. Rev., 128, 803

Davidsson, B. J. R., Sierks, H., Guettler, C., et al. 2016, A\&A, 592, A63

Dominik, C., \& Tielens, A. 1997, ApJ, 480, 647

Ellerbroek, L. E., Gundlach, B., Landeck, A., et al. 2017, MNRAS, 469, S204

Fulle, M., \& Blum, J. 2017, MNRAS, 469, S39

Fulle, M., Levasseur-Regourd, A. C., McBride, N., \& Hadamcik, E. 2000, ApJ, 119, 1968

Fulle, M., Della Corte, V., Rotundi, A., et al. 2015, ApJ, 802, L12

Fulle, M., Marzari, F., Della Corte, V., et al. 2016, ApJ, 821, 19

Fulle, M., Della Corte, V., Rotundi, A., et al. 2017, MNRAS, 469, S45

Güttler, C., Mannel, T., Rotundi, A., et al. 2019, A\&A, 630, A24 (Rosetta 2 SI)

Hamaker, H. C. 1937, Physica, 4, 1058

Hornung, K., Merouane, S., Hilchenbach, M., et al. 2016, Planet. Space Sci., 133, 63

Hörz, F., Bastien, R., Borg, J., et al. 2006, Science, 314, 1716

Israelachvili, J. N. 2011, Intermolecular and Surface Forces (Cambridge: Academic press)

Jullien, R., \& Botet, R. 1987, Ann. Telecommun., 41, 343

Kempf, S., Pfalzner, S., \& Henning, T. K. 1999, Icarus, 141, 388

Kimura, H. 2001, J. Quant. Spectr. Rad. Transf., 70, 581
Kissel, J., Altwegg, K., Clark, B. C., et al. 2007, Space Sci. Rev., 128, 823

Langevin, Y., Hilchenbach, M., Ligier, N., et al. 2016, Icarus, 271, 76

Langevin, Y., Hilchenbach, M., Vincendon, M., et al. 2017, MNRAS, 469, S535

Lasue, J., Levasseur-Regourd, A. C., Hadamcik, E., \& Alcouffe, G. 2009, Icarus, 199, 129

Levasseur-Regourd, A.-C., Zolensky, M., \& Lasue, J. 2008, Planet. Space Sci., 56,1719

Levasseur-Regourd, A.-C., Agarwal, J., Cottin, H., et al. 2018, Space Sci. Rev., 214, 64

Lorensen, W. E., \& Cline, H. E. 1987, ACM Siggraph Computer Graphics (New York: ACM), 21, 163

Mannel, T., Bentley, M. S., Schmied, R., et al. 2016, MNRAS, 462, S304

Meakin, P. 1991, Rev. Geophys., 29, 317

Merouane, S., Zaprudin, B., Stenzel, O., et al. 2016, A\&A, 596, A87

Riedler, W., Torkar, K., Jeszenszky, H., et al. 2007, Space Sci. Rev., 128, 869

Rotundi, A., Sierks, H., Della Corte, V., et al. 2015, Science, 347, aaa3905

Weidenschilling, S. J. 1997, Icarus, 127, 290

Wurm, G., \& Blum, J. 1998, Icarus, 132, 125 